\title{
IoT Enabled Solar Smart Inverter
}

\author{
John Francis P, Abhinav A.P., Jeffin Mathew, Edwin Mathew
}

\begin{abstract}
Solar smart inverters are inverters that are charged through solar power and can be controlled and monitored using IoT. The notion of power in the modern world has become instrumental for the progress of any economy. Almost all engines that run a civilisation like homes, enterprises and governments now rely on energy to ensure appropriate functioning. The exponential rise in population coupled with massive pollution from conventional energy resources has led to enormous demand for green power and enhanced pressure on utility grids. For these reasons the future of the energy industry lies in energy generation from renewable sources and efficient energy storage. Energy storage is a convenient backup tool to use against unforeseen circumstances like breakdowns, floods and storms that thwart vital power supply for uncertain time durations. The constraint of increasing population has also complicated global power systems with power cuts and power shortages. But with huge progress in IT sector, most electrical appliances such as inverters are bound to be smarter with time. This smartness is induced in it by allowing information flow between user and machine.

We intend to create an IoT-enabled solar smart inverter that uses Wi-Fi technology to engage a two-way communication with the user and equipment. The battery SoC, electrolyte level, and run time of the load on the battery are communicated to the user. The user can control the connected load wirelessly employing a mobile application. This will enable the efficient utilization of energy and also upgrades human comfort. An Arduino Uno with Node MCU which runs on the ESP8266 Wi-Fi module is often used to implement the aforementioned objectives. The output power from solar panel can be maximized by making use of an MPPT system, to obtain high power.
\end{abstract}

Index Terms-Battery Health Monitoring System, Integrated Development Environment, IoT, Maximum Power Point Tracking.

\section{INTRODUCTION}

Solar smart inverters are generally defined as inverters that are fed with solar electric energy and can be monitored and controlled by the user. Power has become very much crucial in the proper functioning of a modern economy.

John Francis P., Department of Electrical and Electronics Engineering, APJ Abdul Kalam Technological University, Rajagiri School of Engineering and Technology, Ernakulam, India

Abhinav A. P., Department of Electrical and Electronics Engineering, APJ Abdul Kalam Technological University, Rajagiri School of Engineering and Technology, Ernakulam, India

Jeffin Mathew, Department of Electrical and Electronics Engineering, APJ Abdul Kalam Technological University, Rajagiri School of Engineering and Technology, Ernakulam, India

Edwin Mathew, Department of Electrical and Electronics Engineering, APJ Abdul Kalam Technological University, Rajagiri School of Engineering and Technology, Ernakulam, India
The engines of a civilization such as enterprises, homes and even government entities find it hard to operate without access to uninterrupted energy supplies. The exponential rise in population since the $21^{\text {st }}$ century has created an imbalance in energy supply and demand. Pollution from primitive and conventional energy resources has skyrocketed since the dawn of this century. Hence, it's crucial that we initiate switching over to renewable energy sources to reduce the dependence on conventionally polluting energy sources and so it has become inevitable to put more onus on renewable energy generation and efficient energy storage techniques to dampen the existent pressure on conventional power grids. Energy storage devices offer great convenience during power supply interruption scenarios such as energy failures, floods and storms. The population surge has also had a detrimental effect in power shortages and resultant power cuts. With technical and technological evolution in IT, more electrical and electronic appliances such as even an inverter are bound to be smarter. This smartness allows information to be shared between user and machine.

Here the focus is on monitoring the inverter's DC source and remote control of loads. Inverters used for basic applications in industries or homes commonly runs on non-renewable energy resources and are very much elementary in how they are designed and used. Conventional consumers are often caught off-guard when the battery feeding the inverter fails due to numerous reasons as conventional inverters are handicapped when it comes to sharing its operational information. However, a smart inverter besides using renewable energy to feed itself, also facilitates information sharing in real-time with the consumer. Hence it opens a pool of opportunities in retrofitting conventional inverters to let them be more user-friendly by relaying information on battery charge and battery health, which ultimately promotes the judicious use of obtainable energy by the user.

\section{LITERATURE SURVEY}

Gopal G Menon, Jidhun Das, Sai Kiran, Dr. Siny Paul: This paper proposes an IoT-based solar smart inverter. In this work, a single axis solar tracking mechanism is used to maximize efficiency. It uses a $12 \mathrm{~V}$ battery for storing the generated voltage, which also consists of a battery charge indicator system using a potential divider circuit. For connecting the battery to the loads, a square wave inverter is used. Loads are controlled using NodeMCU based on the ESP8266 wi-fi module.[1]

M. G. Villalva, J. R. Gazoli, E. Ruppert F and Mummadi Veerachary, Jubaer Ahmed, Zainal Salam: shows that "maximum power point tracking mechanism can increase the efficiency of the system by approximately $30 \%$ than 
overcharge controllers that do not implement MPPT. And the frequently used algorithm is the Perturb and Observe (P\&O) technique. The common reason is that the $\mathrm{P} \& \mathrm{O}$ algorithm can be a cheap digital device ensuring robust and good efficiency." [2][3][4]

Hend Abd El-monem Salama, Adel Taha Mohamed Taha: This paper presents solar system development in a lab. "The system under study uses a hard and fast solar module and it's converted into a dual-axis tracking module. A dual-axis tracker can trace the sun vertically and horizontally through optimum light sensors connected to the system. Maximum power point tracking (MPPT) solar model has been incorporated to reduce the cost system of tracking the panel, when compared with other methods of solar tracking systems. A new model tracking system is analyzed and compared with the old fixed system. The control unit is connected to the solar system to implement independent operation of solar tracking or integrated into other solar panels. The results ensure that the MPPT is energy efficient and more reliable than a modified fixed solar panel." [5]

Aishwarya Kumar, Anusha Ashok Bijapur, Charitha B, Kirthi R Kulkarni, K Natarajan: This paper describes the implementation of a smart inverter, i.e., a solar-charged inverter that uses $\mathrm{Wi}-\mathrm{Fi}$ technology is used in a two-way communication with the user, informing the user of both the battery voltage of the inverter and the run time of the load which the user chooses to run. Furthermore, wireless control of loads is implemented to extend user comfort. This paper suggests the use of MSP430F5529LP microcontroller along with an ESP8266 Wi-Fi module.[6]

Kumar Mandula, Ramu Parupalli, CH.A.S. Murty, E. Magesh, Rutul Lunagariya: This paper debates the process of home automation using Bluetooth and Ethernet. When connectivity with a Arduino and smartphone is established using Bluetooth, short-range wireless two-way communication is possible in an indoor environment. Ethernet module is used for connecting Arduino board from any part of the planet.[7]

\section{SYSTEM ARCHITECTURE}

\section{A. Objective}

This project aims to design and develop a solar inverter with efficient solar energy capturing using maximum power point tracking and load control using IoT. The battery charge level, health, electrolyte levels, and battery drain time, are measured using sensors and a potential divider circuit that is connected to an Arduino, and the information is displayed on an LCD display and a cloud website. The inverter is of 500W wattage which converts $12 \mathrm{~V}$ DC to $230 \mathrm{~V}$ sine wave AC signal required for the loads. The output of the inverter is given to a relay connected to NodeMCU for implementing load switching by the user through a mobile application. The Arduino code required for the different features is to be developed. The simulations of main power electronic circuits such as inverter and charge controller are to be done for checking the outputs for further fabrication and assembling of the circuits

\section{B. Basic block diagram of the system}

The basic block diagram of the system is shown below. This block diagram is just a simple outline of the entire system and each block has complex circuits.

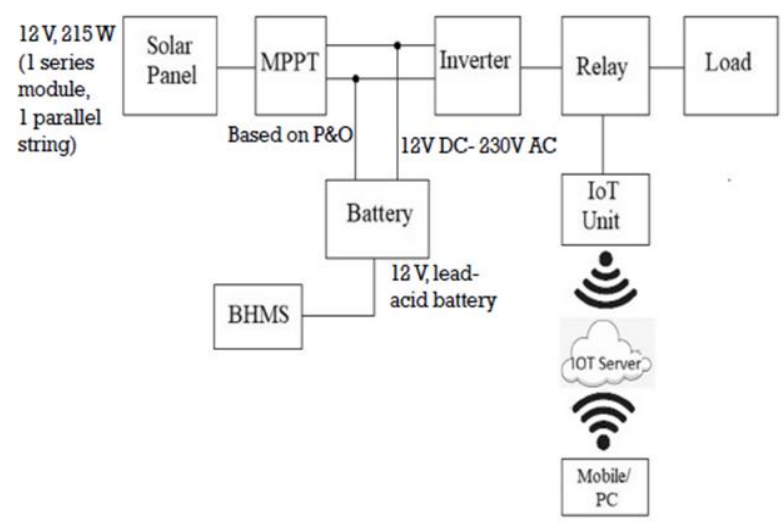

Fig.1. Block diagram of the system

\section{HARDWARE COMPONENTS}

\section{A. Current Sensor}

In the projected system, the current is measured by using ACS712-30A sensor. The sensor gives accurate current measurements. Thus, the power consumption of the overall system can be monitored. The output voltage of the sensor is proportional to the observed current based on the Hall Effect principle. In the ACS712 sensor, 5V supply and ground is connected to the respective terminals of the Arduino. ACS712 module can measure current in AC or DC ranging from $+30 \mathrm{~A}$ to $-30 \mathrm{~A}$. $\mathrm{V}_{\text {out }}$ creates a yield that mirrors the current moving through the detecting component until it is turned up. If the load is turned off, $\mathrm{V}_{\mathrm{cc}} / 2$ will be produced. The ACS712 will measure current in both directions, with a voltage higher than $2.5 \mathrm{~V}$ indicating one direction and a voltage lower than $2.5 \mathrm{~V}$ indicating the other. The analog output of the ACS712 module is read using an analog pin on the Arduino. The figure below shows the ACS712-30A current sensor.

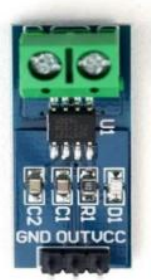

Fig.2. ACS712-30A Current sensor

\section{B. Ultrasonic Sensor}

In this projected system, the sensor used is the HC-SR04, shown in the below figure. In the sensor the ultrasonic transmitter transmits an ultrasonic wave that travels through 
the air and when it comes in contact with any object, the wave gets reflected towards the sensor and this reflected wave is received by the receiver module. The transmitter will emit a $40 \mathrm{kHz}$ ultrasonic pulse, and the receiver will wait for it to return and During this time the echo pin of the sensor will give a high output. The duration of this high output is recorded and is used to calculated the distance between the object and the sensor

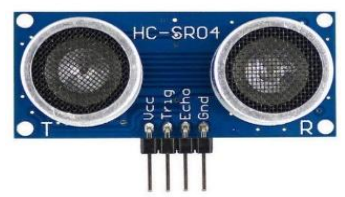

Fig.3. Ultrasonic Sensor

\section{Arduino Uno}

The Arduino Uno is a microcontroller board that uses ATmega328P. It has six analog pins and 14 digital pins and accepts $\mathrm{V}_{\mathrm{CC}}$ from a range of $7 \mathrm{v}$ to $20 \mathrm{v}$. It is programmed using the Arduino IDE software and it can be used for numerous applications.

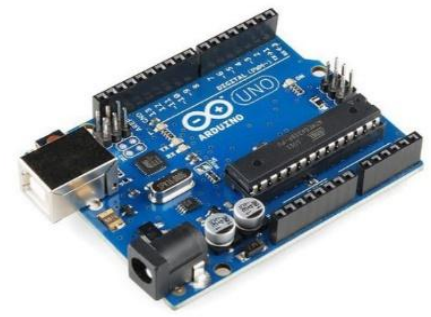

Fig.4. Arduino Uno

\section{Wi-Fi Module}

Wi-Fi module is used for the communication purpose. Its main function is to convert serial port into an embedded module which can be compatible to wireless network communication standard as Wi-Fi. We utilize the ESP8266 $\mathrm{Wi}-\mathrm{Fi}$ module shown in the figure below, as it offers a reliable and complete Wi-Fi networking solution. It can host the appliance or offload all the Wi-Fi networking functions from another application processor. Connectivity is achieved through the UART interface. Performance and memory required is improved through cache. Another advantage of this module is that it is designed to be a compact PCB.

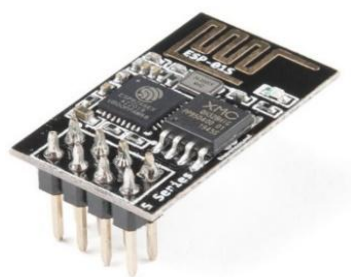

Fig.5. ESP8266 Wi-Fi Module

\section{E. Relay Module}

The relay module is a separate hardware device used for remote device switching. It allows remote control of devices over a network or the web. Devices are often remotely powered on or off, when a pulse is provided to the relay module. Here we are using a 4-channel isolated 5V 10A relay module. A good range of microcontrollers like Arduino, AVR, PIC, ARM can control it. It can also control various appliances and other equipment that runs on high current.

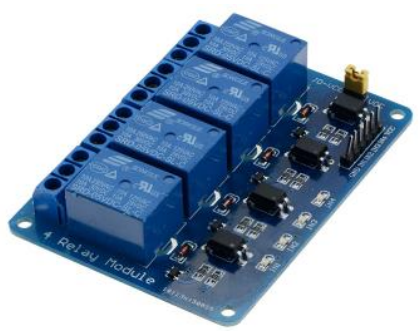

Fig.6. Relay Module

\section{F. Solar Cells}

A solar cell, or photovoltaic cell, is a device that converts the heat energy obtained from sunlight into useful electricity, by means of photovoltaic effect. It is a sort of photoconductive cell, whose electrical characteristics such as current, voltage, resistance vary under sunlight. Individual photovoltaic cells are connected in a combined structure to form modules or solar panels. The common single-junction silicon solar cell can produce a maximum open-circuit voltage of roughly 0.5 volts to 0.6 volts. Solar cells are described as being photovoltaic regardless of whether the source is sunlight or artificial light. In addition to producing energy, they will be used as a photodetector (for example infrared detectors), detecting light or other electromagnetic waves near the visible range or measuring candlepower.

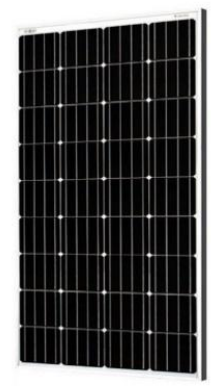

Fig.7. Solar Cell

\section{G. NodeMCU}

NodeMCU is an open source IoT platform, which consists of a firmware, based on the ESP8266 wi-fi module from Espressif systems. It is the central co-coordinator of the sensors and therefore the actuators. This microcontroller has built-in support for Wi-Fi connectivity which allows it to send and receive data from the mobile application via an internet server. It reads sensor data and sends them to the mobile application and receives commands from the mobile application to control home appliances. It then drives the relay module to control the appliances. 


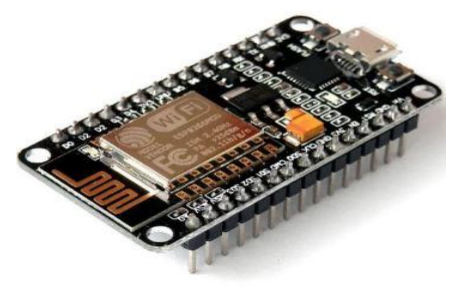

Fig.8. NodeMCU

\section{SOFTWARE REQUIREMENTS}

\section{A. Arduino IDE}

It is an open-source user friendly software used to write and upload codes to any Arduino microcontroller, and it supports a wide range of firmware.

\section{B. Proteus Design Suite}

The Proteus Design Suite is a closed-source development toolkit produced by lab-center mainly used in the electronic design automation. Electronic design engineers and technicians rely on this software to create a simulation of the project to see if it's feasible for production

\section{MATLAB}

MATLAB is a proprietary, high-performance, programming language and computing software used for technical computing. It integrates computation, visualization, and programming in a user-friendly interface consisting of various mathematical notations. Here the MATLAB is used to design the MPPT charge controller circuit and inverter.

\section{ELECTRICAL DESIGN}

\section{A. MPPT Charge Controller}

MPPT addresses one of the main problems of the solar tracking system, which is the efficiency of power transfer from the solar cell depending on the amount of sunlight falling on the solar panels, the temperature of the solar panel, and the electrical characteristics of the load. As these conditions vary, the load characteristic that provides the best power transfer efficiency also changes. The efficiency of the system is optimized when the load characteristic changes to stay the facility transfer at the very best efficiency. This load characteristic is known as the Maximum Power Point (MPP). MPPT is the process used to find this point and keep the load characteristic at that point for maximum transfer efficiency. Solar cells have a posh relationship between temperature and total resistance that produces a non-linear output efficiency which may be analysed supported by the I-V curve. The MPPT system matches the output of the PV cells with appropriate load to obtain maximum power for any given environmental conditions. MPPT devices are usually integrated into electric power converters.

Perturb and Observe algorithm: In this method, the controller adjusts the voltage by very small incremental amounts from the array and measures the power; if power increases, suitable adjustments are made, and the cycle is repeated until power no longer increases. This is called the perturb and observe method. Perturb and Observe is mostly used in MPPT systems due to its reduced expenses, complexity and easier implementation

\section{B. Inverter}

An inverter converts a DC voltage into an AC voltage of variable magnitude and variable frequency. The power semiconductor device which is used in the inverter for switching operation is a MOSFET. MOSFETS guarantee high switching frequency as they are fully controlled devices. A sawtooth carrier waveform of high frequency is compared with a sinusoidal modulating signal of $50 \mathrm{~Hz}$ to generate a SPWM waveform. This waveform is then fed in tandem using a logical NOT operator to gate terminals of MOSFETS $1 \& 2$ and MOSFETS $3 \& 4$ which operate as switches in pairs exclusive of each other. When these switches receive drive signals, they switch between ON and OFF states at rates of $50 \mathrm{~Hz}$. This switching action causes a $50 \mathrm{~Hz}$ sinusoidal AC current to start circulating through the LC filter. The passband of the filter is chosen appropriately to allow the fundamental component to pass through restricting harmonics. This sinusoidal signal goes through a step-up transformer to elevate voltage levels up to $230 \mathrm{~V}$. A purely resistive load is connected at the transformer secondary to run simulations.

\section{IoT and load switching}

The IoT section enables the consumer to control the loads using his/her smartphone. In order to establish this section, we use NodeMCU, an open- source IoT platform, based on the ESP8266 wi-fi module. The loads are connected via the relay module, which is interfaced with the NodeMCU. On the consumer side, we use a mobile application known as Blynk, which processes the requests on its own servers. When a specific load is to be switched on, a virtual switch created on the application will be engaged by the consumer, which generates a signal to be sent to the IoT servers. This signal is processed by the NodeMCU, and the appropriate relay channel is operated, thus turning $\mathrm{ON}$ the load.

\section{Battery Health Monitoring System}

The battery is one of the most expensive components in a solar inverter system, so it must be maintained with care. The battery health monitoring system is capable of measuring the charge percentage, electrolyte levels, and discharge time of a battery and it will alert the user if the battery is damaged or if the battery health is poor, or if any parameter is lower than standard levels. In a BHMS, we use a potential divider circuit to reduce the voltage to a range of 0 to $5 \mathrm{~V}$, and it's fed to an MCP3008 analog to digital converter.

Since the Arduino has only one analog to digital converter it cannot read multiple analog values simultaneously. The MCP3008 reads the analog values of voltage and the Acs712. This value is used to calculate the terminal voltage and soc of the battery and just from the terminal voltage, we can check the battery health, i.e., for a $12 \mathrm{~V}$ battery if the terminal voltage is less than $10.5 \mathrm{~V}$ the battery will no longer function properly so the Arduino is programmed to monitor this and the ultrasonic sensor is used to check the electrolyte levels and if the levels are lower than the predetermined level it will alert the user. The current measured using the current sensor is used to calculate the remaining discharge time to let the 
user know how long the backup power will last with his current load capacity and all this information is displayed through a Wi-Fi module on to the thinkspeak IoT platform and it can be accessed by the user from any computer or smartphone device.

\section{SIMULATIONS AND RESULTS}

\section{A. MPPT Charge Controller}

The Simulink model of the MPPT charge controller was designed using MATLAB software.

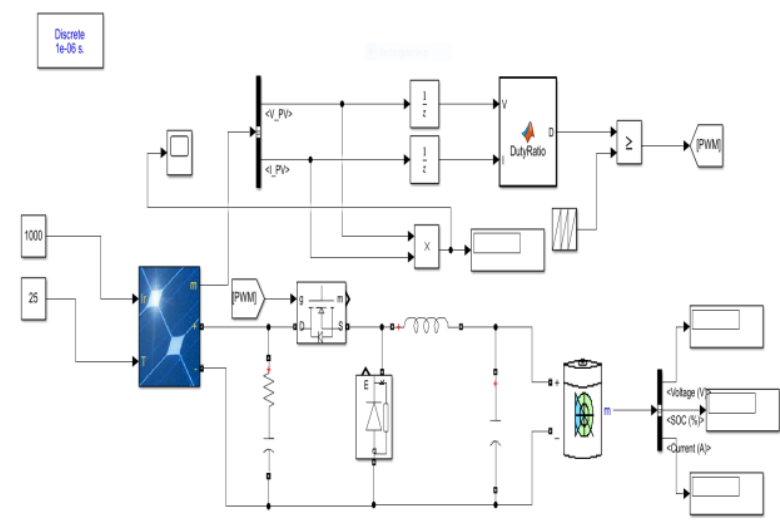

Fig.9. MPPT Charge Controller

The following graphs shows the current vs voltage and power vs voltage characteristics for the selected solar panel in MATLAB software. The dots in theses characteristics indicates the MPP for various values of irradiation level.

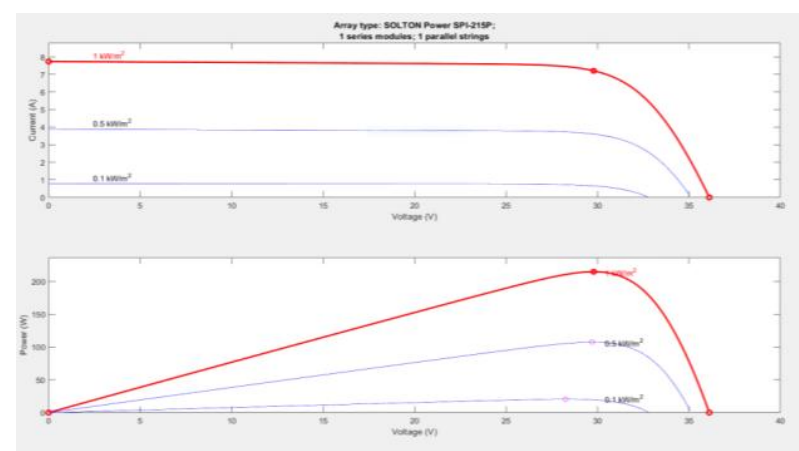

Fig.10. Current vs Voltage and Power vs Voltage characteristics showing MPP

After a few initial oscillations, the MPPT attains maximum power point as shown below.

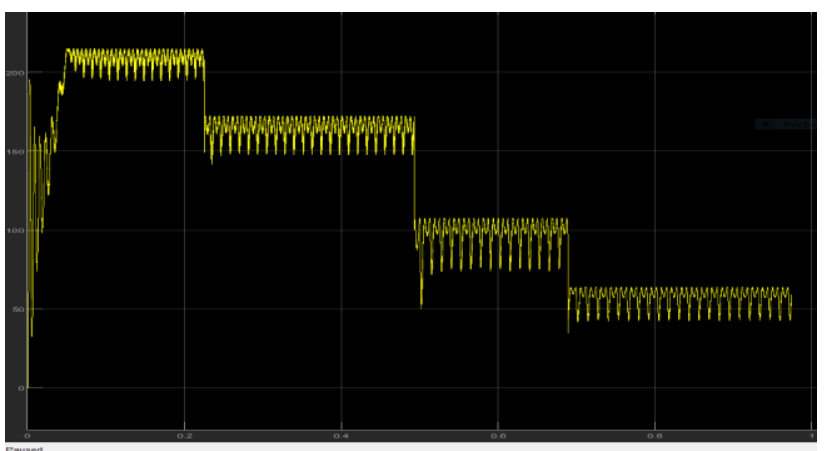

Fig.11. Power vs Time graph

The above graph shows different values of MPP at different irradiation levels.

\section{B. IoT and Load Switching}

The simulation parameters for the IoT and Load Switching is given in the table:

\begin{tabular}{|l|l|}
\hline PARAMETER & SPECIFICATION \\
\hline Lamp & $12 \mathrm{~V} * 2$ \\
\hline Relay & $5 \mathrm{~V} * 2$ \\
\hline Arduino Uno & Arduino Uno R3*1 \\
\hline \multicolumn{2}{|l|}{ Table.1. Simulation parameters for IoT and load switching }
\end{tabular}

A circuit is formed using the parameters mentioned, and is simulated in PROTEUS as below:

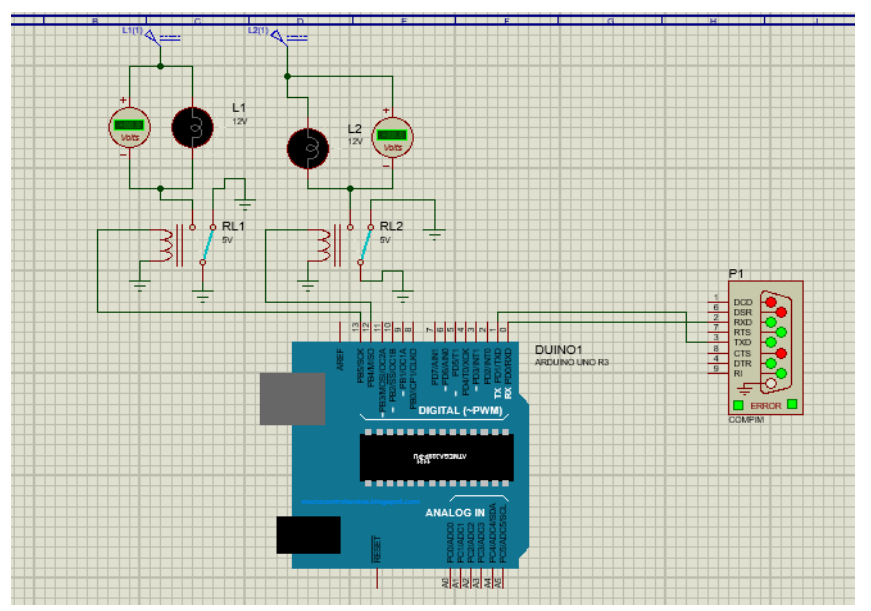

Fig.11. IoT and Load Switching

\section{Battery Health Monitoring System}

The simulation parameters for the BHMS are given in the table:

\begin{tabular}{|l|l|}
\hline PARAMETER & SPECIFICATION \\
\hline LCD Display & $16 \mathrm{x} 2$ \\
\hline Resistors & $6.7 \mathrm{~K}, 3.78 \mathrm{~K}, 650 \mathrm{ohm}$ \\
\hline Arduino Uno & Arduino Uno R3 \\
\hline Rheostat & $10 \mathrm{k}, 1 \mathrm{k}$ ohm \\
\hline Ultrasonic sensor & HC-SR04 \\
\hline
\end{tabular}


IoT Enabled Solar Smart Inverter

\begin{tabular}{|l|l|}
\hline ADC converter & MCP3008 \\
\hline Current Sensor & ACS712 \\
\hline
\end{tabular}

Table.2. Simulation parameters for BHMS

A circuit is formed using the parameters mentioned, and is simulated in PROTEUS as below.

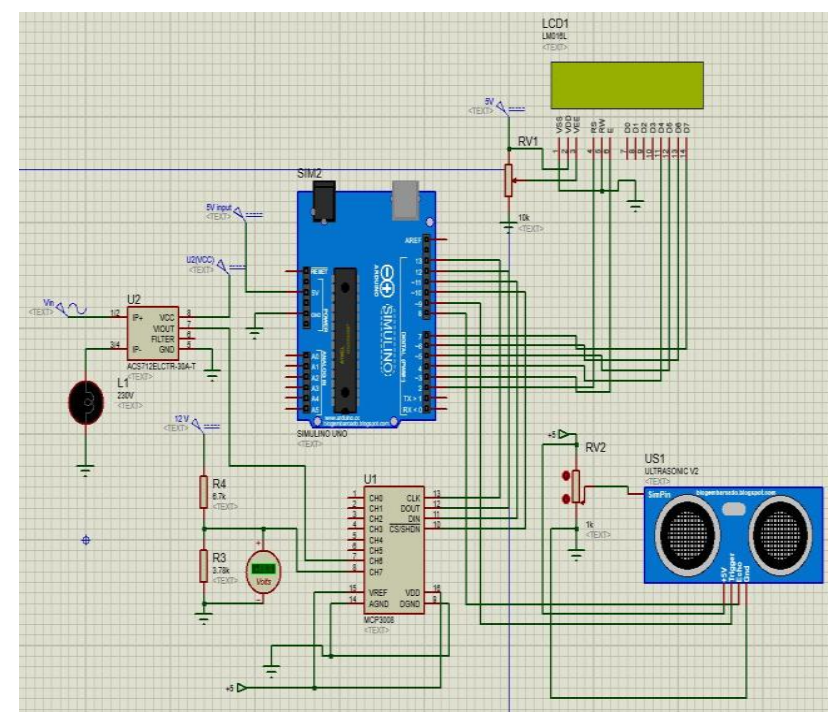

Fig.12. Battery Health Monitoring System

Since the Wi-Fi module is not available in the Proteus Simulation to show the working of the $\mathrm{Wi}-\mathrm{Fi}$ module in the system a Similar Circuit with just the electrolyte detector was created in the thinker-CAD online simulating platform.

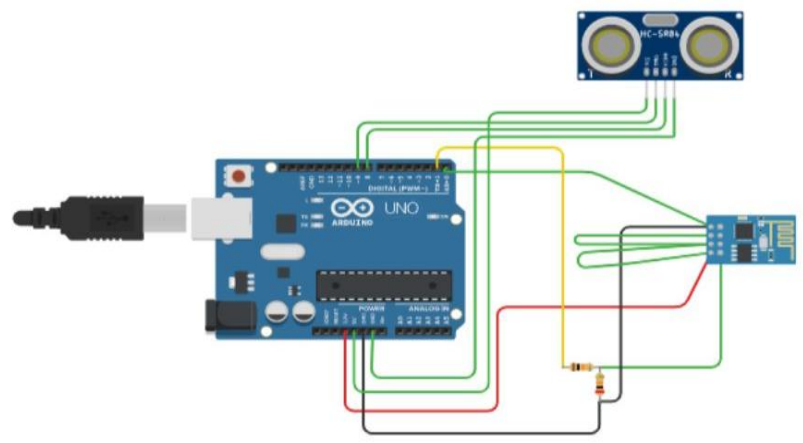

Fig.13. Thinker-CAD model for BHMS

The result is displayed on the ThingSpeak website and the electrolyte level is displayed in percentage in a gauge meter as shown in the figure below:

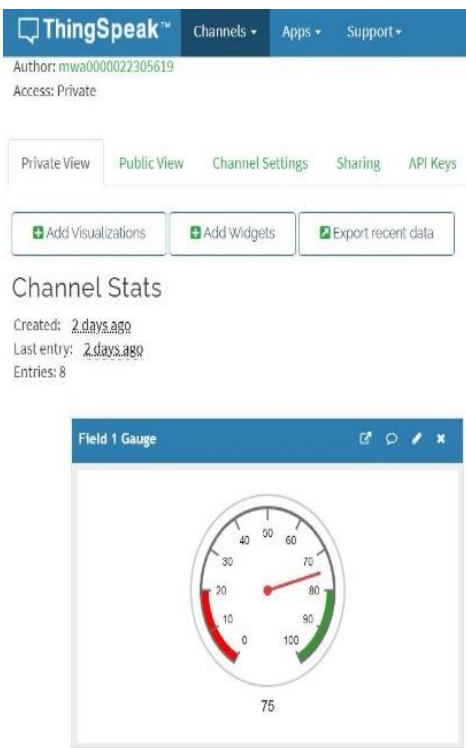

Fig.14. The electrolyte level in ThingSpeak

\section{Inverter}

The Simulink model of a full bridge pure sinewave inverter was designed using MATLAB software.

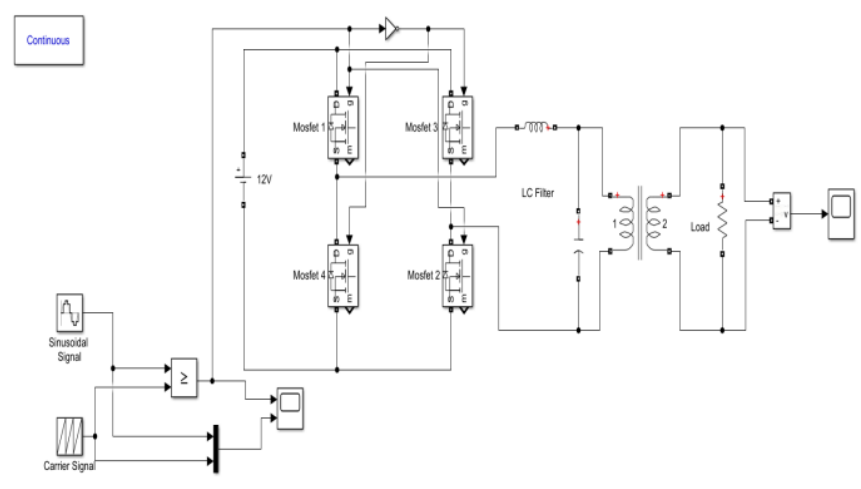

Fig.15. Full Bridge Inverter

The simulations performed on a sample load that was connected across the secondary of the transformer gave pure sinusoidal waveform as its output voltage.

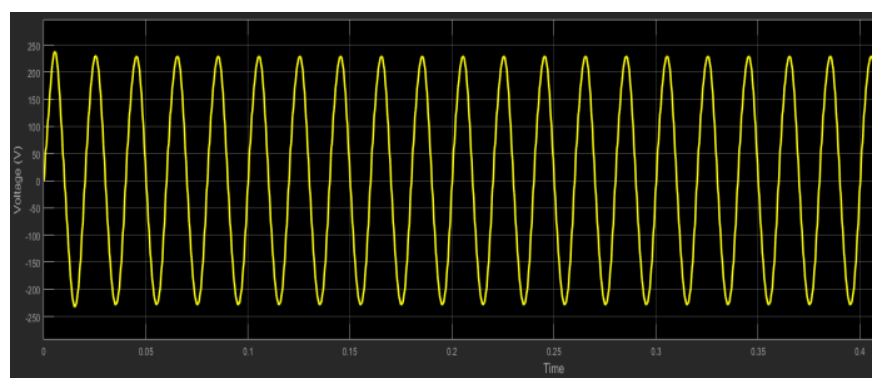

Fig.16. Output voltage across sample load

\section{CONCLUSION}

In this project, we successfully designed a Solar Smart Inverter with load control via IoT. The simulations of the inverter were done in MATLAB and the respective waveforms of output were obtained. The battery health monitoring system and IoT load switching were done in Proteus, and the proper working of the circuits was verified. 
The programming for both of these circuits was also completed. The MPPT can give a maximum power of $215 \mathrm{~W}$. The inverter can give $230 \mathrm{~V}$ output and can handle $800 \mathrm{VA}$. The battery Health monitoring system can measure the real-time state of charge indication and is capable to monitor battery health, electrolyte levels, and full discharge time, the data was displayed on a website. The load control using the IoT unit and Blynk app worked as intended.

\section{REFERENCES}

[1] Gopal G Menon, Jidhun Das, Sai Kiran, Dr. Siny Paul, "Solar Smart Inverter", International Journal of Engineering and Technology (IRJET), vol 07, no. 4, April 2020

[2] M. G. Villalva, J. R. Gazoli, E. Ruppert F, "Modelling and circuit-based simulation of photovoltaic arrays", Brazilian Journal of Power Electronics, 2009 vol. 14 , no. 1 , pp. $35--45$, ISSN 1414-8862.

[3] Mummadi Veerachary, "Control of TI-SEPIC Converter for Optimal Utilization of PV Power", IICPE, 2010 New Delhi.

[4] Jubaer Ahmed, Zainal Salam, "An improved perturb and observe (P\&O) maximum power point tracking (MPPT) algorithm for higher efficiency", Applied Energy, Vol. 150, pp. 97-108, 2015.

[5] Hend Abd El-monem Salama, Adel Taha Mohamed Taha, "Practical Implementation of Dual Axis Solar Power Tracking System", Twentieth International Middle East Power Systems Conference (MEPCON), 2018.

[6] Aishwarya Kumar, Anusha Ashok Bijapur, Charitha B, Kirthi R Kulkarni, K Natarajan, "An IOT based smart inverter", IEEE International Conference on Recent Trends in Electronics, Information \& Communication Technology (RTEICT), 2016.

[7] Kumar Mandula, Ramu Parupalli, CH.A.S. Murty, E. Magesh, Rutul Lunagariya, "Mobile based home automation using Internet of Things (IoT)", International Conference on Control, Instrumentation, Communication and Computational Technologies (ICCICCT), 2015. 